\title{
On a class of abstract convex cone valued functional equations
}

\author{
JUSTYNA SIKORSKA@
}

Dedicated to Professor János Aczél on the occasion of his 95th birthday.

\begin{abstract}
We present an approach to solving a number of functional equations for functions with values in abstract convex cones. Such cones seem to be good generalizations of, e.g., families of nonempty compact and convex subsets or nonempty closed, bounded and convex subsets of a normed space. Moreover, we study some related stability problems.
\end{abstract}

Mathematics Subject Classification. 39B52, 39B82, 54C60, 26E25.

Keywords. Abstract convex cone, Additive function, Quadratic function, Drygas equation, Set-valued function, Stability.

\section{Introduction}

In $[14]$ we solve the functional equation

$$
\frac{\beta+1}{2 \beta^{2}} f(\beta x)=\frac{\beta-1}{2 \beta^{2}} f(-\beta x)+f(x)
$$

for an integer $\beta>1$, where $f$ is a function from a group uniquely divisible by $\beta$ to a family of nonempty compact and convex subsets of a locally convex linear metric space with an invariant metric. The form of solutions of this single variable equation helped us to find solutions of a number of set-valued functional equations in several variables.

The idea for studying (1) and all the computations leading to this equation are described in [11]. There are also several applications concerning stability considerations presented there. The ranges of functions in the mentioned papers were Banach spaces (which in fact could easily be generalized to complete linear metric spaces or sequentially complete linear topological spaces) or hyperspaces of nonempty compact and convex subsets of a locally convex linear metric space. 
A natural question, particularly after studying the latter case, is whether similar results can be obtained for functions with values in arbitrary semigroups.

In the present paper we solve (1) for functions with values in abstract convex cones which seem to be a good generalization of a family of nonempty compact and convex subsets of a normed space.

Besides questions concerning solutions of (1), one may state questions connected with the approximation of a function satisfying (1) or its stability. In the paper, we address a question about the stability of (1) and its equivalent form.

\section{Preliminaries}

Let $(S,+)$ be a commutative semigroup with zero satisfying the cancelation law:

$$
(s+u=t+u \Rightarrow s=t) \text { for all } s, t, u \in S .
$$

We call $S$ an abstract convex cone if a mapping $(\lambda, s) \mapsto \lambda s$ from $[0, \infty) \times S$ into $S$ satisfies the conditions

$$
\lambda(\mu s)=(\lambda \mu) s, \quad \lambda(s+t)=\lambda s+\lambda t, \quad(\lambda+\mu) s=\lambda s+\mu s, \quad 1 \cdot s=s
$$

for all $s, t \in S$ and $\lambda, \mu \geqslant 0$ (see, e.g., $[3,10]$ ).

We will consider an abstract convex cone with a metric $\varrho: S \times S \rightarrow[0, \infty)$. We say further that $\varrho$ is translation invariant or positively homogeneous provided that

$$
\varrho(s+u, t+u)=\varrho(s, t) \text { for all } s, t, u \in S,
$$

or

$$
\varrho(\lambda s, \lambda t)=\lambda \varrho(s, t) \text { for all } s, t \in S, \lambda>0,
$$

respectively.

In 1952, Rådström [10] showed that such a cone can be embedded isometrically and isomorphically into a normed space.

Examples of abstract convex cones:

$1^{\circ}$ an arbitrary normed vector space $(X,\|\cdot\|)$ with the induced metric $\varrho(s, t)=\|s-t\|, s, t \in X$, and the operations + and $\cdot$ from X. If $S \subset X$ is a convex cone (i.e., $s+t, \lambda s \in S$ for all $s, t \in S$ and $\lambda \geqslant 0$ ) then $S$ is an abstract convex cone. It is complete whenever $X$ is a Banach space and $S$ is closed in $X$;

$2^{\circ}$ a collection of nonempty, compact and convex subsets of a real normed linear space $Y$ (denoted $c c(Y)$ ) with the Hausdorff distance and operations:

$$
A+B=\{a+b: a \in A, b \in B\}, \quad \lambda A=\{\lambda a: a \in A\}
$$


(for some details see, e.g., [2]);

$3^{\circ}$ a collection of nonempty, closed, bounded and convex subsets of a real normed linear space $Y$ (denoted $b c l(Y)$ ) with the Hausdorff distance and operations:

$$
A \stackrel{*}{+} B=\operatorname{cl}(A+B), \quad \lambda A=\{\lambda a: a \in A\} .
$$

Let $S$ be a semigroup with the cancelation law. For elements $s, t, u \in S$ we say that $u$ is an abstract $S$-difference of $s$ and $t$, i.e., $u=s-t$ if and only if $s=u+t$. If this difference exists, then it is unique. In the space $c c(Y)$ (see $2^{\circ}$ above) such a difference is known as the Hukuhara difference (see [6]).

The following lemmas will be useful in the next part of the paper.

Lemma 2.1. Let $S$ be an abstract convex cone and let $\varrho$ be a translation invariant, positively homogeneous and complete metric on $S$. Assume that for $s, t, s_{n}, t_{n} \in S, n \in \mathbb{N}$, we have $s_{n} \rightarrow s, t_{n} \rightarrow t$ and for every $n \in \mathbb{N}$ there exists an abstract $S$-difference $s_{n}-t_{n}$. Then there exists an abstract $S$-difference $s-t$ and $s_{n}-t_{n} \rightarrow s-t$.

The proof of the above lemma goes along the same lines as in [9, Lemma 1]. In particular cases of abstract convex cones we do not need to assume the completeness of the space (see [14, Lemma 2.6] for the case of the family of nonempty, compact and convex subsets of a locally convex linear metric space with an invariant metric). Also, if our cone is a closed proper subset of a metric space, we obtain the same assertion as in Lemma 2.1.

Lemma 2.2. Let $(X, \varrho)$ be a metric space and let $S \varsubsetneqq X$ be an abstract convex cone with the metric inherited from $X$, which is translation invariant and positively homogeneous in $S$. Assume that the cone $S$ is closed in $X$. If for $s, t, s_{n}, t_{n} \in S, n \in \mathbb{N}$, we have $s_{n} \rightarrow s, t_{n} \rightarrow t$ and for every $n \in \mathbb{N}$ there exists an abstract $S$-difference $s_{n}-t_{n}$, then there exists an abstract $S$-difference $s-t$ and $s_{n}-t_{n} \rightarrow s-t$.

Proof. By the assumptions, for every $n \in \mathbb{N}$ there exists $u_{n}:=s_{n}-t_{n}$ which means that $s_{n}=u_{n}+t_{n}$. We have

$$
\varrho\left(u_{n}+t, s\right)=\varrho\left(u_{n}+t_{n}+t, s+t_{n}\right)=\varrho\left(s_{n}+t, s+t_{n}\right) \leqslant \varrho\left(s_{n}, s\right)+\varrho\left(t, t_{n}\right),
$$

whence,

$$
u_{n}+t \rightarrow s
$$

that is, the sequence $\left(u_{n}+t\right)_{n \in \mathbb{N}}$ is convergent in $S$. On the other side, $u_{n}+t \in$ $S+t$ for all $n \in \mathbb{N}$ and $s \in S+t$, since $S+t$ is closed. Consequently, $s=u+t$ for some $u \in S$, there exists the abstract $S$-difference $s-t$ and, finally, $u_{n} \rightarrow u$.

Remark 2.1. It is worth observing that the abstract convex cone $c c(Y)$, where $Y$ is a normed linear space, is closed in $b c l(Y)$ or in the space of all nonempty subsets of $Y$ (cf., Lemma 2.6 in [14]). 
The following easy example shows that we may not omit the assumption about the completeness or closedness of $S$.

Example 2.1. Take an abstract convex cone $S=\left\{(x, y) \in \mathbb{R}^{2}: x, y>0\right\} \cup$ $\{(0,0)\}$ or $S=\left\{(x, y) \in \mathbb{R}^{2}: x, y \geqslant 0\right.$ and $\left.(x=0 \Rightarrow y=0)\right\}$ with the usual addition and scalar multiplication and with the euclidean distance, and define $s_{n}=\left(1+\frac{2}{n}, 2+\frac{2}{n}\right)$ and $t_{n}=\left(1+\frac{1}{n}, 1+\frac{1}{n}\right)$ for $n \in \mathbb{N}$. We have $s=(1,2) \in S$, $t=(1,1) \in S$, and $s_{n}-t_{n}=\left(\frac{1}{n}, 1+\frac{1}{n}\right) \in S, n \in \mathbb{N}$, but there does not exist $s-t$ in $S$.

\section{Solutions of the equation}

We start this section with presenting the main result concerning solutions of the equation in a single variable introduced at the beginning of the paper.

Theorem 3.1. Let $\beta$ be a positive integer different from $1,(G,+)$ be a commutative group uniquely divisible by $\beta$, and let $S$ be an abstract convex cone with a translation invariant, positively homogeneous and complete metric. Assume that $f: G \rightarrow S$ satisfies (1). Then there exist uniquely determined functions $a, q: G \rightarrow S$ such that $f=a+q$,

$$
a(x)+a(-x)=0, x \in G, \text { and } q \text { is even. }
$$

Moreover,

$$
a(\beta x)=\beta a(x), \quad q(\beta x)=\beta^{2} q(x), \quad x \in G .
$$

Conversely, each function $f$ of the form $a+q$, where $a, q: G \rightarrow S$ are such that (2) and (3) hold, satisfies (1).

Proof. By induction, we get from (1)

$$
\frac{\beta^{n}+1}{2 \beta^{2 n}} f\left(\beta^{n} x\right)=\frac{\beta^{n}-1}{2 \beta^{2 n}} f\left(-\beta^{n} x\right)+f(x), \quad x \in G, n \in \mathbb{N},
$$

that is,

$$
\frac{\beta^{n}\left(\beta^{n}+1\right)}{2 \beta^{2 n}} f(x)=\frac{\beta^{n}\left(\beta^{n}-1\right)}{2 \beta^{2 n}} f(-x)+\beta^{n} f\left(\frac{x}{\beta^{n}}\right), \quad x \in G, n \in \mathbb{N} .
$$

From Lemma 2.1 we derive that for every $x \in G$ there exists the abstract $S$-difference $\frac{1}{2} f(x)-\frac{1}{2} f(-x)$. Define $a(x):=\frac{1}{2} f(x)-\frac{1}{2} f(-x), x \in G$. Then

$$
\frac{1}{2} f(x)=a(x)+\frac{1}{2} f(-x)=a(x)+a(-x)+\frac{1}{2} f(x),
$$

so $a(x)+a(-x)=0$. We also have

$$
a(x)+\frac{1}{2} f(-x)+\frac{1}{2} f(x)=\frac{1}{2} f(x)+\frac{1}{2} f(x)=f(x) .
$$


If now we define $q(x):=\frac{1}{2} f(x)+\frac{1}{2} f(-x)$ for all $x \in G$ then $f(x)=a(x)+$ $q(x), x \in G$, and $q$ is even.

Both $a$ and $q$ satisfy (1), whence $a(\beta x)=\beta a(x)$ and $q(\beta x)=\beta^{2} q(x)$.

The proof of the converse is immediate.

Remark 3.1. Instead of the completeness of $S$ in Theorem 3.1 (and in the following Theorem 3.2), on account of Lemma 2.2 we may assume that $S$ is a proper closed subset of a metric space.

Theorem 3.1 brings a number of immediate applications in solving equations in several variables for abstract convex cone-valued functions. We present here a few of them (cf., e.g., $[4,7,8,12,14,15])$ :

(a) the Drygas equation: $f(x+y)+f(x-y)=2 f(x)+f(y)+f(-y)$ (Theorem 3.1 applied with $\beta=2)$;

(b) the Fréchet equation: $f(x+y+z)+f(x)+f(y)+f(z)=f(x+y)+$ $f(y+z)+f(z+x)(\beta=2)$

(c) the equation of orthogonal additivity: $x \perp y \Rightarrow f(x+y)=f(x)+f(y)$ $(\beta=2)$;

(d) $9 f\left(\frac{x+y+z}{3}\right)+f(x)+f(y)+f(z)=4\left[f\left(\frac{x+y}{2}\right)+f\left(\frac{y+z}{2}\right)+f\left(\frac{z+x}{2}\right)\right](\beta=$ $3)$;

(e) $f(x+\beta y)+\beta f(x-y)=f(x-\beta y)+\beta f(x+y)(\operatorname{arbitrary} \beta)$;

(f) $f(2 x+y)+f(2 x-y)+2 f(x)=f(x+y)+f(x-y)+2 f(2 x)(\beta=2)$.

In all the above examples we obtain solutions of the form $f=a+q$ (in (d)-(f) up to a constant) with $a$ and $q$ having properties as in the assertion of the mentioned theorem. In the last three cases we assume additionally that for every $x \in G$ there exists the abstract $S$-difference $f(x)-f(0)$. The forms of equations (a)-(f) imply additionally that $a$ is additive (i.e., $a(x+y)=a(x)+$ $a(y)$ for all $x, y \in G)$ and $q$ is quadratic (i.e., $q(x+y)+q(x-y)=2 q(x)+2 q(y)$ for all $x, y \in G$ ).

As an example we show such an application for the Drygas equation.

Theorem 3.2. Let $(G,+)$ be a commutative group uniquely divisible by 2 , and let $S$ be an abstract convex cone with a translation invariant, positively homogeneous and complete metric. Assume that $f: G \rightarrow S$ satisfies

$$
f(x+y)+f(x-y)=2 f(x)+f(y)+f(-y), \quad x, y \in G .
$$

Then there exist an additive function $a: G \rightarrow S$ and a quadratic function $q: G \rightarrow S$ such that $f=a+q$ and such a representation is unique. Moreover, each function of the form $a+q$, where $a: G \rightarrow S$ is additive and $q: G \rightarrow S$ is quadratic, is a solution of (4).

Proof. Setting $y=x$ in (4) we get

$$
f(2 x)=3 f(x)+f(-x), \quad x \in G .
$$


Small rearrangements lead to

$$
\frac{3}{8} f(2 x)=f(x)+\frac{1}{8} f(-2 x), \quad x \in G,
$$

which means that (1) is satisfied with $\beta=2$. By Theorem 3.1, there exist functions $a, q: G \rightarrow S$ such that $f=a+q$ and

$$
\begin{aligned}
& a(2 x)=2 a(x), \quad a(x)+a(-x)=0, \quad x \in G, \\
& q(2 x)=4 q(x), \quad q(x)=q(-x), \quad x \in G .
\end{aligned}
$$

Using the form of $f$ in (4), writing it also for $-x$ instead of $x$ and $-y$ instead of $y$, and finally adding both equations side by side, we conclude that $q$ is quadratic, and consequently, $a$ satisfies

$$
a(x+y)+a(x-y)=2 a(x), \quad x, y \in G .
$$

Since $a(0)=0$, it follows from the above that $a$ is additive. The rest is proved in a standard way.

\section{Stability considerations}

In what follows we answer the question whether for a function $f: G \rightarrow S$ satisfying inequality

$$
\varrho\left(f(x)+\frac{\beta-1}{2 \beta^{2}} f(-\beta x), \frac{\beta+1}{2 \beta^{2}} f(\beta x)\right) \leqslant \varphi(x), \quad x \in G,
$$

with some control function $\varphi: G \rightarrow[0, \infty)$, there exists a function $g: G \rightarrow S$ which satisfies (1) and which is close (with respect to the metric $\varrho$ ) to $f$. In case of a positive answer we say that (1) is stable (the reader interested in learning more on stability is referred, e.g., to $[1,5])$.

We will show, however, that without any additional assumption imposed on the class of functions for which (5) is considered, the equation is not stable (cf., [13]).

To see this, consider $S=c c(\mathbb{R})$, the family of nonempty, closed and bounded intervals. It is easy to see that for $A=\left[\alpha_{1}, \alpha_{2}\right], B=\left[\beta_{1}, \beta_{2}\right]$ the Hausdorff distance

$$
\varrho(A, B)=\max \left\{\left|\alpha_{1}-\beta_{1}\right|,\left|\alpha_{2}-\beta_{2}\right|\right\} .
$$

Consequently, if $f: G \rightarrow c c(\mathbb{R})$ is of the form

$$
f(x)=[p(x), q(x)], \quad x \in G
$$

for some functions $p, q: G \rightarrow \mathbb{R}, p \leqslant q$, then the condition

$$
\varrho\left(f(x)+\frac{\beta-1}{2 \beta^{2}} f(-\beta x), \frac{\beta+1}{2 \beta^{2}} f(\beta x)\right) \leqslant \varepsilon,
$$


with some $\varepsilon \geqslant 0$ (i.e., we have (5) in the simplest case where $\varphi(x) \equiv \varepsilon$ ), is equivalent to the system of conditions

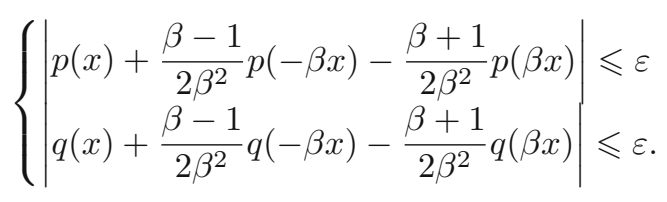

Example 4.1. Fix $\varepsilon>0$ and define functions $p, q: \mathbb{R} \rightarrow \mathbb{R}$,

$$
p(x)=\frac{1}{2} \varepsilon\left(x^{2}+x+1\right)>0 \text { and } q(x)=\varepsilon\left(x^{2}+x+1\right)>0 .
$$

Then $p<q$ and functions $p$ and $q$ satisfy system (8). Consider $f: \mathbb{R} \rightarrow c c(\mathbb{R})$ of the form (6). As a consequence, $f$ satisfies (7).

However, there does not exist a function $g: \mathbb{R} \rightarrow c c(\mathbb{R})$ satisfying (1) and such that

$$
\varrho(f(x), g(x)) \leqslant M, \quad x \in \mathbb{R},
$$

with some constant $M$ depending on $\varepsilon$. Indeed, suppose to the contrary that such $g$ does exist. Then $g=a+b$, where $a: \mathbb{R} \rightarrow c c(\mathbb{R})$ is such that $a(x)+$ $a(-x)=\{0\}$ for all $x \in \mathbb{R}$ and $b: \mathbb{R} \rightarrow c c(\mathbb{R})$ is even. It follows that $a$ is single-valued and odd. If we denote $b=\left[b_{1}, b_{2}\right]$ with $b_{1}, b_{2}: \mathbb{R} \rightarrow \mathbb{R}, b_{1} \leqslant b_{2}$, then $g(x)=\left[a(x)+b_{1}(x), a(x)+b_{2}(x)\right]$ for all $x \in \mathbb{R}$.

It follows from (8) and [11, Corollary 2.2] that there exist uniquely determined functions $\widetilde{p}, \widetilde{q}: \mathbb{R} \rightarrow \mathbb{R}$ satisfying (1) and such that

$$
|p(x)-\widetilde{p}(x)| \leqslant \frac{\beta \varepsilon}{\beta-1} \quad \text { and } \quad|q(x)-\widetilde{q}(x)| \leqslant \frac{\beta \varepsilon}{\beta-1}
$$

for all $x \in \mathbb{R}$. It is easy to verify that in our case $\widetilde{p}(x)=\frac{1}{2} \varepsilon\left(x^{2}+x\right)$ and $\widetilde{q}(x)=\varepsilon\left(x^{2}+x\right)$ for all $x \in \mathbb{R}$.

Since the function

$$
\varrho(f(x), g(x))=\max \left\{\left|p(x)-a(x)-b_{1}(x)\right|,\left|q(x)-a(x)-b_{2}(x)\right|\right\}, \quad x \in \mathbb{R}
$$

is bounded, by the uniqueness of $\widetilde{p}$ and $\widetilde{q}$, it follows that

$$
\widetilde{p}(x)=a(x)+b_{1}(x) \text { and } \widetilde{q}(x)=a(x)+b_{2}(x) \text { for all } x \in \mathbb{R} .
$$

This is impossible since the odd parts of $\widetilde{p}$ and $\widetilde{q}$ equal to $\frac{1}{2} \varepsilon x$ and $\varepsilon x$, respectively, are different.

Remark 4.1. The above non-stability result shows that, in general, all the (sometimes called 'additive-quadratic' in the literature) functional equations in several variables for functions with values in abstract convex cones, while considering the control function $\varphi(x) \equiv \varepsilon$ or, more generally, $\varphi$ satisfying $\sum_{n=0}^{\infty} \frac{1}{\beta^{n}} \varphi\left(\beta^{n} x\right)<\infty$ (with $\beta>1$ ), are not stable either. 
As we have just seen, (1) is in general not stable. However, considering again $S=c c(\mathbb{R})$ and making some additional assumptions on $p$ and $q$, we obtain some positive stability results.

Theorem 4.1. Let $\beta$ be a positive integer different from $1,(G,+)$ be a group uniquely divisible by $\beta$, and let $f: G \rightarrow c c(\mathbb{R})$ of the form (6) satisfy (7) with some $\varepsilon>0$. If $q(x)-p(x)>\frac{2 \beta \varepsilon}{\beta-1}$ for all $x \in G \backslash\{0\}$, then there exists a unique function $g: G \rightarrow c c(\mathbb{R})$ satisfying (1) and such that

$$
\varrho(f(x), g(x)) \leqslant \frac{\beta \varepsilon}{\beta-1}, \quad x \in G .
$$

Proof. By the assumption, functions $p$ and $q$ satisfy (8) and from [11, Corollary 2.2] it follows that there exist functions $\widetilde{p}, \widetilde{q}: G \rightarrow \mathbb{R}$ which satisfy (1) and (9) for all $x \in G$. As a result,

$$
\widetilde{p}(x) \leqslant p(x)+\frac{\beta \varepsilon}{\beta-1}<q(x)-\frac{\beta \varepsilon}{\beta-1} \leqslant \widetilde{q}(x), \quad x \in G \backslash\{0\} .
$$

Since $\widetilde{p}(0)=\widetilde{q}(0)=0, \widetilde{p}(x) \leqslant \widetilde{q}(x)$ for all $x \in G$.

Surely, the function $g: G \rightarrow c c(\mathbb{R})$ defined by

$$
g(x):=[\widetilde{p}(x), \widetilde{q}(x)], \quad x \in G,
$$

satisfies (1) and, moreover,

$$
\varrho(f(x), g(x))=\max \{|p(x)-\widetilde{p}(x)|,|q(x)-\widetilde{q}(x)|\} \leqslant \frac{\beta \varepsilon}{\beta-1}, \quad x \in G .
$$

To prove the uniqueness, let $\widetilde{g}$ be another function satisfying (1) and such that the (Hausdorff) distance between $f$ and $\widetilde{g}$ is bounded, that is, $\varrho(f(x), \widetilde{g}(x)) \leqslant$ $M$ for all $x \in G$ and some $M>0$. Hence,

$$
\varrho(g(x), \widetilde{g}(x)) \leqslant \frac{\beta \varepsilon}{\beta-1}+M, \quad x \in G .
$$

By Theorem 3.1, $g=a_{1}+b_{1}$ and $\widetilde{g}=a_{2}+b_{2}$ with some functions $a_{1}, a_{2}: G \rightarrow$ $c c(\mathbb{R})$ such that $a_{i}(\beta x)=\beta a_{i}(x)$ for $i \in\{1,2\}$, and $b_{1}, b_{2}: G \rightarrow c c(\mathbb{R})$ such that $b_{i}(\beta x)=\beta^{2} b_{i}(x)$ for $i \in\{1,2\}$. Therefore,

$$
\varrho\left(a_{1}(x)+b_{1}(x), a_{2}(x)+b_{2}(x)\right) \leqslant \frac{\beta \varepsilon}{\beta-1}+M, \quad x \in G,
$$

and

$$
\begin{aligned}
& \varrho\left(a_{1}\left(\beta^{n} x\right)+b_{1}\left(\beta^{n} x\right), a_{2}\left(\beta^{n} x\right)+b_{2}\left(\beta^{n} x\right)\right) \leqslant \frac{\beta \varepsilon}{\beta-1}+M, \quad x \in G, n \in \mathbb{N}, \\
& \varrho\left(\beta^{n} a_{1}(x)+\beta^{2 n} b_{1}(x), \beta^{n} a_{2}(x)+\beta^{2 n} b_{2}(x)\right) \leqslant \frac{\beta \varepsilon}{\beta-1}+M, \quad x \in G, n \in \mathbb{N} .
\end{aligned}
$$

Dividing by $\beta^{2 n}$ and letting $n$ tend to infinity we obtain $b_{1}=b_{2}$. Now, it is easy to see that $a_{1}=a_{2}$, and the proof is complete. 
Theorem 4.2. Let $\beta$ be a positive integer different from $1,(G,+)$ be a group uniquely divisible by $\beta$, and let $f: G \rightarrow c c(\mathbb{R})$ of the form (6) satisfy (7) with some $\varepsilon \geqslant 0$. If $M:=\sup \left\{\left|q_{o}(x)-p_{o}(x)\right|: x \in G\right\}<\infty$, where $p_{o}$, $q_{o}$ stand for the odd parts of $p$ and $q$, respectively, then there exists a uniquely determined function $g: G \rightarrow c c(\mathbb{R})$ satisfying (1) and such that

$$
\varrho(f(x), g(x)) \leqslant \frac{\beta \varepsilon}{\beta-1}, \quad x \in G .
$$

Proof. On account of (8), $d_{o}:=q_{o}-p_{o}$ satisfies

$$
\left|d_{o}(x)+\frac{\beta-1}{2 \beta^{2}} d_{o}(-\beta x)-\frac{\beta+1}{2 \beta^{2}} d_{o}(\beta x)\right| \leqslant 2 \varepsilon, \quad x \in G .
$$

Moreover,

$$
\left|d_{o}(x)\right| \leqslant M, \quad x \in G .
$$

By [11, Corollary 2.2], there exists a uniquely determined function $\widetilde{d}_{o}$ satisfying (1) and such that

$$
\left|d_{o}(x)-\widetilde{d}_{o}(x)\right| \leqslant \frac{2 \beta \varepsilon}{\beta-1}, \quad x \in G .
$$

In fact, function $\widetilde{d}_{o}$ is given by (see [12, Corollary 2.1, Remark 2.3])

$$
\widetilde{d}_{o}(x)=\lim _{n \rightarrow \infty}\left(\frac{\beta^{n}+1}{2 \cdot \beta^{2 n}} d_{o}\left(\beta^{n} x\right)-\frac{\beta^{n}-1}{2 \cdot \beta^{2 n}} d_{o}\left(-\beta^{n} x\right)\right), \quad x \in G,
$$

which on account of $(10)$ yields $\widetilde{d}_{o}=0$. Since $\widetilde{d}_{o}$ is uniquely determined, we have $\widetilde{p_{o}}=\widetilde{q_{o}}$, where $\widetilde{p_{o}}$ and $\widetilde{q_{o}}$ are approximations for $p_{o}$ and $q_{o}$, respectively.

The even parts $p_{e}, q_{e}$ of $p$ and $q$, respectively, satisfy (8) and since $p(x) \leqslant$ $q(x)$, we also have $p_{e}(x) \leqslant q_{e}(x)$ for all $x \in G$. Hence, their even counterparts $\widetilde{p_{e}}$ and $\widetilde{q_{e}}$ satisfy $(1)$ and

$$
\widetilde{p_{e}}(x)=\lim _{n \rightarrow \infty} \frac{1}{\beta^{2 n}} p_{e}\left(\beta^{n} x\right) \leqslant \lim _{n \rightarrow \infty} \frac{1}{\beta^{2 n}} q_{e}\left(\beta^{n} x\right)=\widetilde{q_{e}}(x), \quad x \in G .
$$

Consequently, the function $G \ni x \mapsto b(x):=\left[\widetilde{p_{e}}(x), \widetilde{q_{e}}(x)\right]$, satisfies $(1)$.

Define $g(x):=\left\{\widetilde{p_{o}}(x)\right\}+b(x)$ for all $x \in G$. Then $g$ satisfies (1) and on account of the uniqueness of approximations, we have $\varrho(f(x), g(x))=\varrho\left([p(x), q(x)],\left[\widetilde{p_{o}}(x)+\widetilde{p_{e}}(x), \widetilde{q_{o}}(x)+\widetilde{q_{e}}(x)\right]\right) \leqslant \frac{\beta \varepsilon}{\beta-1}, \quad x \in G$.

For the uniqueness of $g$, we use the same argument as in the proof of the previous theorem.

Assume $(G,+)$ is a group uniquely divisible by $\beta$. Substitute $\frac{x}{\beta}$ and $-\frac{x}{\beta}$ in the place of $x$ in (1). After small rearrangements we get

$$
f(x)=\frac{\beta^{2}+\beta}{2} f\left(\frac{x}{\beta}\right)+\frac{\beta^{2}-\beta}{2} f\left(\frac{-x}{\beta}\right) .
$$


We have seen that Eq. (1) is, in general, not stable. However, in what follows we will show a positive stability result for its equivalent form (12).

Theorem 4.3. Let $\beta$ be a positive integer different from $1,(G,+)$ be a group uniquely divisible by $\beta$, let $S$ be an abstract convex cone with a translation invariant, positively homogeneous and complete metric $\varrho$ and let $\varphi: G \rightarrow[0, \infty)$ be such that $\sum_{n=0}^{\infty} \beta^{2 n} \varphi\left(\frac{x}{\beta^{n}}\right)$ is convergent for all $x \in G$. Assume that $f: G \rightarrow$ $S$ satisfies

$$
\varrho\left(f(x), \frac{\beta^{2}+\beta}{2} f\left(\frac{x}{\beta}\right)+\frac{\beta^{2}-\beta}{2} f\left(\frac{-x}{\beta}\right)\right) \leqslant \varphi(x), \quad x \in G .
$$

Then there exists a uniquely determined function $g: G \rightarrow S$ satisfying (12) and such that

$$
\varrho(f(x), g(x)) \leqslant \sum_{n=0}^{\infty}\left[\frac{\beta^{2 n}+\beta^{n}}{2} \varphi\left(\frac{x}{\beta^{n}}\right)+\frac{\beta^{2 n}-\beta^{n}}{2} \varphi\left(\frac{-x}{\beta^{n}}\right)\right], \quad x \in G .
$$

Proof. By induction, for all $x \in G$ and $n \in \mathbb{N}$,

$$
\begin{gathered}
\varrho\left(f(x), \frac{\beta^{2 n}+\beta^{n}}{2} f\left(\frac{x}{\beta^{n}}\right)+\frac{\beta^{2 n}-\beta^{n}}{2} f\left(\frac{-x}{\beta^{n}}\right)\right) \\
\leqslant \sum_{i=0}^{n-1}\left[\frac{\beta^{2 i}+\beta^{i}}{2} \varphi\left(\frac{x}{\beta^{i}}\right)+\frac{\beta^{2 i}-\beta^{i}}{2} \varphi\left(\frac{-x}{\beta^{i}}\right)\right]
\end{gathered}
$$

Define

$$
g_{n}(x):=\frac{\beta^{2 n}+\beta^{n}}{2} f\left(\frac{x}{\beta^{n}}\right)+\frac{\beta^{2 n}-\beta^{n}}{2} f\left(\frac{-x}{\beta^{n}}\right), \quad x \in G, n \in \mathbb{N} .
$$

It is easy to see that $\left(g_{n}\right)_{n \in \mathbb{N}}$ satisfies the Cauchy condition, and so, it is convergent in $S$. It is enough now to define $g(x):=\lim _{n \rightarrow \infty} g_{n}(x)$ for all $x \in G$ and the rest of the proof goes in a standard way.

Remark 4.2. By Theorem 4.3 we can prove that all functional equations in several variables from which we get a single variable functional equation of the form (12), while considering the control function $\varphi$ such that $\sum_{n=0}^{\infty} \beta^{2 n} \varphi\left(\frac{x}{\beta^{n}}\right)<$ $\infty$, are stable (cf., Remark 4.1).

We finish the paper with the following.

Problem 4.1. The metric structure of an abstract convex cone while looking for the solutions of (1) does not seem to be natural. Is it possible to avoid this assumption? 
Open Access. This article is distributed under the terms of the Creative Commons Attribution 4.0 International License (http://creativecommons.org/licenses/by/4.0/), which permits unrestricted use, distribution, and reproduction in any medium, provided you give appropriate credit to the original author(s) and the source, provide a link to the Creative Commons license, and indicate if changes were made.

Publisher's Note Springer Nature remains neutral with regard to jurisdictional claims in published maps and institutional affiliations.

\section{References}

[1] Brillouët-Belluot, N., Brzdek, J., Ciepliński, K.: On some recent developments in Ulam's type stability. Abstr. Appl. Anal., Art. ID 716936, 41 pp (2012)

[2] Castaing, C., Valadier, M.: Convex Analysis and Measurable Multifunctions. Springer, Berlin (1977)

[3] Chistyakov, V.: Metric Modular Spaces. Springer, Cham (2015)

[4] Ebanks, B.R., Kannappan, P., Sahoo, P.K.: A common generalization of functional equations characterizing normed and quasi-inner product spaces. Canad. Math. Bull 35(3), 321-327 (1992)

[5] Hyers, D.H., Isac, G., Rassias, T.M.: Stability of Functional Equations in Several Variables. Birkhäuser, Boston (1998)

[6] Hukuhara, M.: Intégration des applications measurables dont la valeur est un compact convexe. Funkcial. Ekvac. 10, 205-223 (1967)

[7] Kannappan, P.: Functional Equations and Inequalities with Applications. Springer Monographs in Mathematics. Springer, New York (2009)

[8] Najati, A., Moghimi, M.B.: Stability of a functional equation deriving from quadratic and additive functions in quasi-Banach spaces. J. Math. Anal. Appl. 337, 399-415 (2008)

[9] Piszczek, M.: Second Hukuhara's derivative and cosine family of linear set valued functions. Ann. Acad. Paed. Cracov. Studia Math. 5, 87-98 (2006)

[10] Rådström, H.: An embedding theorem for spaces of convex set. Proc. Am. Math. Soc. 3, 165-169 (1952)

[11] Sikorska, J.: On a direct method for proving the Hyers-Ulam stability of functional equations. J. Math. Anal. Appl. 372, 99-109 (2010)

[12] Sikorska, J.: Set-valued orthogonal additivity. Set-Valued Var. Anal. 23, 547-557 (2015)

[13] Sikorska, J.: A singular behaviour of a set-valued approximate orthogonal additivity. Results Math. 70, 163-172 (2016)

[14] Sikorska, J.: On a method of solving some functional equations for set-valued functions. Set-Valued Var. Anal. 27, 295-304 (2019)

[15] Smajdor, W.: On set-valued solutions of a functional equation of Drygas. Aequationes Math. 77, 89-97 (2009)

Justyna Sikorska

Institute of Mathematics

University of Silesia

Bankowa 14

40-007 Katowice

Poland

e-mail: justyna.sikorska@us.edu.pl

Received: March 27, 2019

Revised: July 24, 2019 